\title{
Source monitoring for actions in adolescents with 22q11.2 deletion syndrome (22q11DS)
}

\author{
M. Debbané ${ }^{1,2 *}$, M. Van der Linden ${ }^{1,2}$, B. Glaser ${ }^{1,2}$ and S. Eliez ${ }^{1,3}$ \\ ${ }^{1}$ Service Médico-Pédagogique Research Unit, Department of Psychiatry, University of Geneva School of Medicine, Switzerland \\ ${ }^{2}$ Faculty of Psychology, University of Geneva, Switzerland \\ ${ }^{3}$ Department of Genetic Medicine and Development, University of Geneva School of Medicine, Switzerland
}

\begin{abstract}
Background. Source monitoring consists in identifying the origin of mental events. Recent research suggests that confusions over internally generated mental events may represent a cognitive marker for increased proneness to psychotic symptoms and disorders. We have examined source monitoring for actions in adolescents with the 22q11.2 deletion syndrome (22q11DS), a neurogenetic disease associated with high rates of schizophrenia during adulthood, and expected to observe source monitoring deficits in comparison to IQ-matched and typically developing controls.
\end{abstract}

Method. Eighteen adolescents with 22q11DS, 17 adolescents matched for age and IQ, and also 17 adolescents matched for age participated in this study. Our adapted action monitoring paradigm asked subjects to visualize a series of actions in three different conditions: (1) visualize themselves performing the action; (2) visualize the experimenter performing the action; or (3) simply repeat the action statements without visualization of the action performer.

Results. The adolescents with 22q11DS performed adequately in terms of recognition (hits), but in comparison to both control groups, they committed more source confusions on correctly recognized items. Further examination revealed that the adolescents were more likely to demonstrate confusions between exterior sources in which the self was not involved.

Conclusions. Source monitoring deficits can be observed in adolescents with 22q11DS, a syndrome putting them at high risk for developing schizophrenia. These deficits are discussed in terms of early cognitive processes associated with genetic risk for schizophrenia.

Received 15 May 2007; Revised 20 September 2007; Accepted 11 October 2007; First published online 16 November 2007

Key words: Memory, schizophrenia, self-monitoring, VCFS.

\section{Introduction}

The 22q11.2 deletion syndrome (22q11DS), also known as velo-cardio-facial syndrome (VCFS), is a neurogenetic syndrome most commonly resulting from a congenital microdeletion on the long arm of chromosome 22. Its prevalence is approximately one in 4300-7000 live births (Oskarsdottir et al. 2004). Psychotic symptoms such as auditory hallucinations can appear during childhood (Debbané et al. 2006a), and half of the adolescents with the syndrome report having experienced positive symptoms (Baker \& Skuse, 2005). Almost $30 \%$ of affected individuals will meet the diagnosis of schizophrenia during adulthood (Murphy et al. 1999), making 22q11DS a potent risk factor for the development of schizophreniform

* Address for correspondence: M. Debbané, Service MédicoPédagogique, Department of Psychiatry, University of Geneva School of Medicine, 16-18 Boulevard St-Georges, Case Postale 50, 1211 Geneva 8, Switzerland.

(Email: martin.debbane@medecine.unige.ch) disorders in adulthood (Bassett et al. 2003; Murphy, 2005).

Very little is known about the cognitive characteristics that underlie risk for schizophrenia in 22q11DS. Cognitive development in this syndrome may include learning difficulties (Swillen et al. 1999), speech and language difficulties (Moss et al. 1999; Glaser et al. 2002), and below-average IQ (Golding-Kushner et al. 1985; Swillen et al. 1997). Verbal and executive deficits in youngsters with the deletion resemble those of other groups that at high risk for schizophrenia (Cornblatt et al. 1999). Two studies suggest that a decrease in verbal IQ accompanies the onset of positive symptoms in adolescents with 22q11DS (Gothelf et al. 2005; Debbané et al. 2006a), and one study suggests that executive function deficits in affected children and adolescents could reflect genetic risk for schizophrenia (Lewandowski et al. 2006). Although these studies inform us about the cognitive similarities between adolescents with 22q11DS and high risk for schizophrenia adolescents, finer cognitive testing such as 
that performed in research on schizophrenia may provide specific information about the actual cognitive processes that contribute to the development and/or maintenance of positive symptoms such as delusions and hallucinations. Cognitive scientists studying schizophrenia have focused on the relationship between hallucinations and source monitoring mechanisms (Bentall et al. 1991; Frith, 1992; Brébion et al. 2005). Source monitoring refers to the processes by which we can successfully infer the origin of mental events such as memories (Johnson et al. 1993). Because memories are created through the binding of features into a more or less distinct event, we require monitoring processes that will infer the exact origin or source of this event (Johnson \& Raye, 2000). Current accounts of source monitoring deficits in schizophrenia suggest that the process of attributing a source to internally generated material (such as thoughts, memories or voluntary actions) is prone to confusion between internal and external sources (Bentall et al. 1994; Franck et al. 2000; Brébion et al. 2002). For example, we may put the blame on our partner for not turning off the oven, when in fact we were responsible for leaving it heating. This represents a common example of attributing an external source to an internally generated mental event. A pathological extreme of such misattributions could be, for example, the attribution of internal speech to an external source, thereby constituting an unusual perceptual experience such as 'hearing voices'. The association between source monitoring deficits and positive symptoms comes from studies reporting increased source monitoring confusions in schizophrenic patients who report auditory hallucinations (Allen et al. 2004; Brunelin et al. 2006). Therefore, altered source monitoring could represent a cognitive process underlying the positive symptoms exhibited by patients with schizophrenia.

Traditional source monitoring paradigms involve both the participant and the experimenter generating material that is subsequently used in the recognition and source attribution procedures. For example, Brébion et al. (2000) present a task where the participant and the examiner generate items belonging to categories (e.g. FRUITS). The experimenter verbally produced an example item (e.g. apple), and then showed a picture of a second example (e.g. cherry), after which the participant provided a third example (e.g. grapes), thus constituting a group of three target items (verbal and picture from experimenter, verbal from the participant) for each category. After a 5-min delay, the recognition procedure consisted in attributing the source of production (self versus experimenter) for recognized category items. This task showed that schizophrenic participants with hallucinations demonstrate an increased tendency to report self-produced items as items produced by the experimenter. Misattributions to an external source (experimenter) of internally generated material (selfproduced items) correspond to an external attribution bias; that is, a tendency to attribute the origin of a mental event generated by the self to an external source.

Most external attribution biases observed in schizophrenic patients correspond to confusions between internal and external events, where events produced by the self (internal) are recalled as having been produced by a non-self agent (external). However, patients with schizophrenia may also confuse two types of events both generated within the self (internal-internal confusions) (Franck et al. 2000). A recent study on source monitoring performances in hallucination-prone college students included both internal-external (self versus experimenter) and internal-internal (self versus self) source discrimination in their experimental design (Larøi et al. 2005). Using an original action monitoring task, the authors proposed five conditions in which participants were to study simple action statements such as 'Opening a car door' by either (1) miming the action; (2) watching the experimenter mime the action; (3) imagining themselves performing the action; (4) imagining the experimenter performing the action; or (5) repeating the action statement without miming or imagining the performance of the action. Therefore, conditions 1 and 2 examined possible internal-external confusions, while conditions 3,4 and 5 examined possible internal-internal confusions. Conditions 3-5 introduce another level of analysis in internal-internal source discrimination, which consists in differentiating between 'personal' (imagining myself opening a door) and extra-personal or 'exterior' sources (imagining the experimenter opening a door; repeating the statement opening a door). In the subsequent recognition phase, participants were presented with a list combining the previously studied action statements with new action statements. Upon source attribution of previously studied action statements, the authors found that hallucination-prone participants committed more internal-internal source confusions; that is, they showed an increased tendency to report actions they imagined themselves performing as actions they imagined the experimenter perform. However, they did not show more external attribution errors in the internal-external condition (self versus experimenter), which would relate more closely to source monitoring tasks such as those used by Brébion and colleagues (2000, 2002, 2005) with schizophrenic patients. The internal-internal confusions committed by hallucination-prone students represent another type of external attribution bias, consisting in recalling 
'personal' events (imagining myself opening a door) as 'exterior' events (imagining the experimenter opening a door). This type of external attribution bias resembles the findings by Franck et al. (2000), where silent words (personal events) were more often recalled as overtly read words (exterior events) by schizophrenic patients with hallucinations. Therefore, external attribution biases also concern 'personal' versus 'exterior' sources in self-generated events.

In brief, source attribution confusions in schizophrenia can occur between self-generated and nonself-generated material (internal-external confusions), as well as between two sources of self-generated materials (internal-internal confusions). In the latter, the source closer to the self (personal event) is more prone to an external attribution (recalled as exterior event) (Franck et al. 2000; Larøi et al. 2005). The only study examining source monitoring in non-clinical participants suggests that hallucination proneness is associated with specific internal-internal source confusions (Larøi et al. 2005). However, it is not clear whether source monitoring deficits precede, co-occur or result from positive symptoms. Indeed, studies examining source monitoring deficits are performed with adult populations, involving individuals within or beyond the crucial period of psychotic unfolding. The examination of source monitoring skills during adolescence could provide evidence for a continuum of source monitoring deficits along the clinical symptomatic continuum for psychosis (Johns \& van Os, 2001). In providing important information on source monitoring skills in the early stages of vulnerability to schizophrenia, such investigations may assist in the identification of early cognitive processes that set the stage for more pathological source attributions such as those observed in auditory hallucinations.

The current study describes the first examination of source monitoring in adolescents with 22q11DS, a neurogenetic syndrome associated with very high risk for schizophrenia. Based on the only high-risk study exploring source monitoring in young adults (Larøi et al. 2005), we chose to specifically examine internal-internal source monitoring. We used the corresponding experimental conditions that led highrisk individuals to the most source confusions in Larøi et al.'s (2005) action monitoring task. We therefore adapted our action monitoring paradigm to include only Larøi et al.'s conditions 3-5, in which the material is generated exclusively by the participant. Given the lower intellectual profile associated with 22q11DS, we included comparison participants matched for IQ, age and gender, as well as another group of healthy controls matched only for age and gender. Limiting the cognitive demand as well as testing time with cognitively impaired participants (22q11DS participants and IQ-matched controls) further motivated our decision to focus on internal-internal source monitoring. On the action monitoring task, we first expected that recognition performances from participants with 22q11DS would be comparable to that of both control groups (Debbané et al. in press). Second, given the very high risk for schizophrenia in this population, we expected to find increased source monitoring confusions in adolescents with 22q11DS. Third, following Larøi et al. (2005), we expected to observe an external attribution bias in the 22q11DS group, expressed through an increased tendency to report actions that participants imagine themselves performing as actions they imagined the experimenter perform. Fourth, we explored possible linear correlations between source monitoring confusions and levels of symptom expression quantified through self-rated measures of schizotypy, depression and anxiety.

\section{Method \\ Participants}

Eighteen participants with 22q11DS (22q group), 17 age- and IQ-matched controls (IQcontrol group), and 17 age-matched healthy controls (AGEcontrol group) were enrolled in this study. Participants with 22q11DS (11 females, seven males) were recruited through announcements in parent association newsletters and by word of mouth. Three participants were receiving methylphenidate at time of participation. All members of the IQcontrol group (11 females, six males) were recruited through a child and adolescent out-patient service (Service Médico-Pédagogique) affiliated to the University of Geneva's Psychiatry Department and to the Canton of Geneva Education Department. All members of the AGEcontrol group (nine females, eight males) were screened for neurological and psychiatric disorders. They were recruited through a newsletter distributed at public schools and in the community near the research centre. Written informed consent was obtained from participants and their parents under protocols approved by the Institutional Review Board of the Department of Psychiatry of the University of Geneva Medical School. All participants underwent an intellectual evaluation, using the Wechsler Intelligence Scale for Children-III (WISCIII) short form (Kaufman et al. 1996). Using ageappropriate self-report instruments, all participants were screened for levels of anxiety [Revised Children's Manifest Anxiety Scale (R-CMAS); Turgeon \& Chartrand, 2003; French version, 1999] and depression [Children's Depression Inventory (CDI); SaintLaurent, 1990]. 
To assess psychotic symptoms and their dimensions, subjects filled out the Schizotypal Personality Questionnaire (SPQ; Raine, 1991), translated into French and validated by Dumas et al. (2000). To ensure that all subjects understood the items, a trained clinical psychologist (M.D.) supervised the questionnaire process. The participants were instructed that the questions they did not understand could be left blank and answered during the questionnaire review period performed with the clinical psychologist after they had finished filling out the self-reports. The instrument yields three main factor scores (CognitivePerceptual, Interpersonal and Disorganization) and nine subscale scores (Ideas of Reference, Social Anxiety, Odd Beliefs/Magical Thinking, Unusual Perceptual Experiences, Eccentric/Odd Behaviour and Appearance, No Close Friends, Odd Speech, Constricted Affect, Suspiciousness/Paranoid Ideation). It also lends itself to multiple dimensional analyses in the context of a dimensional approach to schizotypy (Rossi \& Daneluzzo, 2002), and is appropriate for use with adolescents (Axelrod et al. 2001).

\section{Source monitoring task}

Source monitoring was examined using an action monitoring task adapted from Larøi et al. (2005), that excluded the miming conditions and retained the verbal presentation conditions of the original task, as these yield the most misattributions. We therefore retained conditions 3-5 in which the subject imagines the performance of actions in either self, experimenter or verbal repetition conditions. Specifically, participants were asked to mentally visualize actions that involved either themselves or the experimenter. Thus, during the initial study phase, participants were asked to actively imagine the action read by the experimenter, by (1) imagining themselves performing the action, or (2) imagining the experimenter performing the action. To encourage subjects to actively monitor their visualization activity, they were asked to refer to a visual analogue scale from 1 to 5 and rate the degree of difficulty to perform each visualization ( $1=$ easy, $5=$ difficult). A third control condition consisted in (3) mentally repeating the action statement, and judging the degree of difficulty required to silently repeat the statement. Practice trials for each of the three conditions preceded the start of the study phase.

The experimenter read a total of 61 action statements in fixed random order blocks: four blocks of imagine-self items (20 S-items in total), four blocks of imagine-experimenter items (20 E-items in total), and seven blocks of repeat items (21 R-items in total). Each block was preceded by an instruction stating the condition in which visualizations should occur. For example: 'In the next trials, please imagine "the experimenter" performing the actions.' To minimize contamination between conditions, repeat-item blocks were inserted in between imagine-self and imagineexperimenter blocks. The actions that were included in this task were chosen for simplicity, universality and gender neutrality (see Larøi et al. 2005 for more details on action statements). The incidental nature of this procedure was preferred for its naturalistic quality, as source monitoring skills act upon day-to-day mental activity that typically is free from instructions inciting a subject to remember specific events.

After a 10-15-min visuospatial filler task, the recognition phase was introduced by a brief summary of the study phase, reminding to participants of the three conditions in which the action statements were encoded (imagine-self, imagine-experimenter, repeat). The experimenter then read out a list of actions that included all the items from the study phase, as well as new action statements acting as distractors, in a fixed random order. The participant had to recognize whether the action statement belonged to the study phase (recognition test), and for those that did, demonstrate source monitoring skills by attributing the recognized action statements to their respective condition: imagine-self, imagine-experimenter or repeat item.

\section{Statistical analyses}

Simple ANOVAs were performed for all clinical characteristics except the CDI $t$ score (depression score), for which single group comparisons were performed using Mann-Whitney tests. The ANOVAs were followed by post-hoc group comparisons using Tukey's correction to identify significant group differences. One-way ANOVAs were performed to examine group differences on recognition and source monitoring performances. False recognition group comparisons were performed using the KruskalWallis test because of the data's non-parametric distribution. Recognition performances were tested using the Total correct recognition score (hits), which represented the total number of correct recognitions of previously studied action statements. For action statements recognized correctly, source monitoring performances were collected by tabulating the total number of source monitoring confusions for each participant. The Total Confusion score was therefore used to compare source monitoring performances between groups. Total False Recognition scores consisted of the total number of distractor action statements recognized as previously studied action statements. Post-hoc comparisons using Tukey's 
Table 1. Clinical characteristics for each group including mean values, standard deviations and ranges

\begin{tabular}{llll}
\hline & $\begin{array}{l}\text { 22q11DS group } \\
(n=18)^{\mathrm{a}}\end{array}$ & $\begin{array}{l}\text { IQcontrol group } \\
(n=17)\end{array}$ & $\begin{array}{l}\text { AGEcontrol group } \\
(n=17)\end{array}$ \\
\hline Age (years) & $\begin{array}{l}14.04 \pm 1.28 \\
(12.5-17.5)\end{array}$ & $\begin{array}{l}14.76 \pm 1.26 \\
(12.6-16.6)\end{array}$ & $\begin{array}{l}(15.05 \pm 1.58 \\
(79.1-16.9)\end{array}$ \\
FSIQ estimate ${ }^{\mathrm{b}}$ & $\begin{array}{l}7.69 \pm 10.40^{*} \\
(65-100)\end{array}$ & $\begin{array}{l}\left(65.60 \pm 12.64^{*}\right. \\
106.31 \pm 8.68\end{array}$ & $(95-126)$ \\
R-CMAS & $71.17 \pm 27.78$ & $78.65 \pm 23.61$ & $62.71 \pm 31.61$ \\
total score percentile & $(28-99)$ & $(18-99)$ & $(5-99)$ \\
CDI $t$ score & $51.61 \pm 8.94^{*}$ & $58.12 \pm 12.15^{*}$ & $44.82 \pm 5.05$ \\
& $(41-78)$ & $(42-88)$ & $(37-55)$ \\
SPQ total score & $22.22 \pm 12.20$ & $24.24 \pm 10.81^{*}$ & $13.82 \pm 10.14$ \\
& $(8-45)$ & $(2-39)$ & $(0-32)$ \\
SPQ Cognitive-Perceptual & $7.67 \pm 5.86$ & $10.24 \pm 6.27^{*}$ & $5.35 \pm 4.78$ \\
SPQ Interpersonal & $(1-18)$ & $(1-20)$ & $(1-14)$ \\
& $9.56 \pm 4.66^{*}$ & $8.06 \pm 4.24$ & $5.18 \pm 4.19$ \\
SPQ Disorganization & $(3-20)$ & $(0-15)$ & $(0-15)$ \\
& $5.00 \pm 3.09$ & $5.94 \pm 4.28$ & $3.29 \pm 2.85$ \\
\hline
\end{tabular}

FSIQ, Full-scale IQ; R-CMAS, Revised Children's Manifest Anxiety Scale; CDI, Children's Depression Inventory; SPQ,

Schizotypal Personality Questionnaire.

a 22q11DS and IQcontrol groups do not differ significantly on any clinical characteristic measures.

${ }^{\mathrm{b}}$ Estimate derived from the recommended the Wechsler Intelligence Scale for Children-III (WISC-III) short form by Kaufman et al. (1996), which includes Similarities, Arithmetic, Picture Completion and Block Design subtests.

* $p<0.05$ on group comparisons with AGEcontrols.

correction were then performed to identify which experimental condition(s) yielded significant contrasts.

\section{Results}

Group comparisons for clinical characteristics are summarized in Table 1. Globally, the 22q11DS and IQcontrol groups did not differ significantly on any clinical characteristic measure. Significant differences were found between the AGEcontrol group in comparison to both 22q11DS and IQcontrol groups on the depression measure $(z=-2.42, p=0.016 ; z=-3.61$, $p<0.001$, respectively) and on the full-scale IQ (FSIQ) estimate measure $(F=19.1, \mathrm{df}=2, p<0.001)$. Followup Tukey HSD pairwise tests revealed that the 22q11DS group further differed from the AGEcontrol group on the Interpersonal factor score from the SPQ $(p=0.033)$, and the IQcontrol group differed from the AGEcontrol group on the Total SPQ score $(p=0.023)$ and Cognitive-Perceptual factor score $(p=0.04)$.

For performance on the action monitoring paradigm, group comparisons yielded no significant differences on the total number of hits $(F=0.03, \mathrm{df}=2$, $p=0.974)$ or false recognitions $\left(\chi^{2}=4.03, \mathrm{df}=2\right.$, $p=0.13)$. However, significant differences were found on the total confusions score $(F=5.22, \mathrm{df}=2, p=0.009)$. Follow-up Tukey HSD pairwise tests revealed significant group differences on 22q11DS and IQcontrols $(p=0.039)$ and 22q11DS and AGEcontrols $(p=0.012)$ contrasts, indicating that participants with 22q11DS made significantly more source monitoring confusion errors than both comparison groups (Table 2).

We proceeded to examine which experimental condition(s) might account for these significant differences. Individual condition contrasts were based on the number of confusions divided by the number of correct recognitions, yielding an individual source monitoring ratio for each participant. For each condition, there were two possible source monitoring confusions: actions imagined as performed by self but remembered as being performed by the experimenter (self_exp) or repeated (self_rep); actions imagined as performed by the experimenter but remembered as performed by self (exp_self) or repeated (exp_rep); and repeated actions remembered as performed by self (rep_self) or the experimenter (rep_exp). We performed a MANOVA including all six source monitoring confusion ratios, illustrated in Fig. 1. The model yielded an overall group effect $(F=2.64, \mathrm{df}=5$, $p=0.005)$. Follow-up ANOVAs for each confusion 
Table 2. Group performances on action monitoring task in terms of total correct recognitions, total false recognitions and total source monitoring confusions

\begin{tabular}{lccr}
\hline & $\begin{array}{l}\text { 22q11DS group } \\
(n=18)\end{array}$ & $\begin{array}{l}\text { IQcontrol group } \\
(n=17)\end{array}$ & $\begin{array}{l}\text { AGEcontrol group } \\
(n=17)\end{array}$ \\
\hline Total correct recognitions (hits) & $51.44 \pm 7.47$ & $51.76 \pm 6.65$ & $51.24 \pm 6.10$ \\
Total false recognitions & $2.39 \pm 0.90$ & $0.35 \pm 0.17$ & $1.29 \pm 0.55$ \\
Total source monitoring confusions & $19.33 \pm 8.17^{*}$ & $13.29 \pm 5.58$ & $12.18 \pm 7.17$ \\
\hline
\end{tabular}

${ }^{*} p<0.05$ on group comparisons with IQcontrol and AGEcontrol groups.

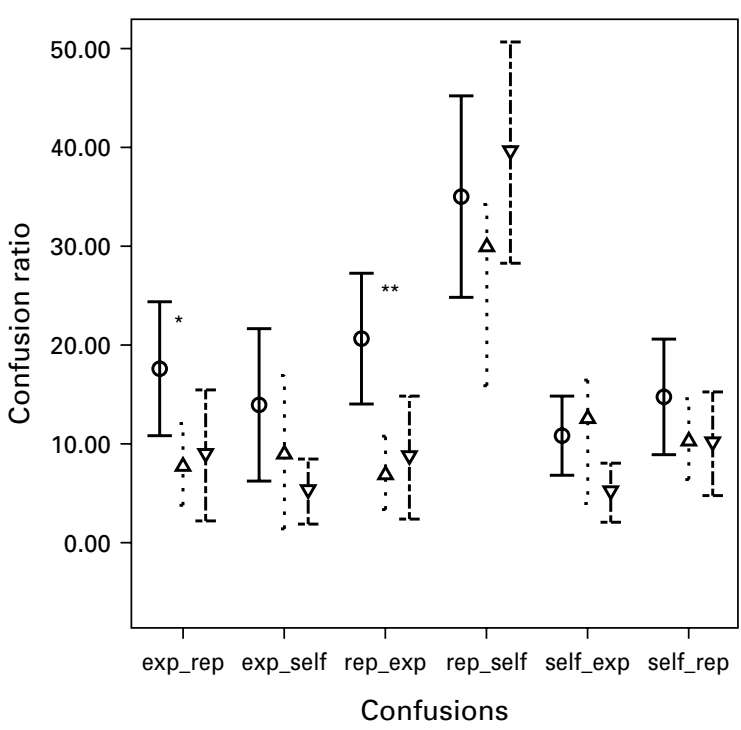

Fig. 1. Groups' confusion ratios for the six source monitoring confusion types. exp_rep, remembering imagineexperimenter items as repeat items; exp_self, remembering imagine-experimenter items as imagine-self items; rep_exp, remembering repeat items as imagine-experimenter items; rep_self, remembering repeat items as imagine-self items; self_exp, remembering imagine-self items as imagineexperimenter items; self_rep, remembering imagine-self items as repeat items. ${ }^{*} 22 \mathrm{q} 11 \mathrm{DS} v$. AGEcontrols $(-\bigcirc-$; $p<0.05) ;{ }^{* *} 22 q 11 D S v$. IQcontrols $(-\nabla-; p<0.01)$ and AGEcontrols $(--\triangle--; p<0.005)$. Error bars show $95 \%$ confidence intervals of the mean.

type indicated significant differences for exp_rep $(F=3.63, \mathrm{df}=2, p=0.034)$ and rep_exp confusions $(F=7.63, \mathrm{df}=2, p=0.001)$. Finally, post-hoc Tukey HSD pairwise tests yielded a significant contrast between 22q11DS and AGEcontrols $(p=0.048)$ for the exp_rep confusions, and two significant contrasts between 22q11DS and both IQcontrols $(p=0.008)$ and AGEcontrols $(p=0.002)$ for the rep_exp contrast. These contrasts indicated that adolescents from the 22q11DS group committed more exp_rep mistakes compared to adolescents from the AGEcontrols group, and more rep_exp mistakes compared to both control groups.
Follow-up correlations were performed to identify any possible relationship between psychological variables and source monitoring performances. Pearson correlations between psychological variables (R-CMAS total percentile score, CDI $t$ score, SPQ total score and SPQ Cognitive-Perceptual score) and source monitoring variables (Total Confusions score, exp_rep ratio and rep_exp ratio) in 22q11DS did not yield any significant relationship $(r<0.31, p>0.23)$.

\section{Discussion}

This study is the first to report source monitoring deficits in adolescents with 22q11DS, a neurogenetic condition associated with elevated risk for schizophrenia. Using an action monitoring paradigm, the results show that the adolescents with 22q11DS presented average recognition performances (hits) but committed more source confusion errors than both IQcontrols and AGEcontrols. Post-hoc analyses revealed that confusion errors mainly occurred in the 'exterior' sources conditions. Specifically, adolescents with 22q11DS committed more source confusions by recalling imagine-experimenter actions as actions they repeated mentally, and vice versa, by recalling repeatactions as actions they imagined the experimenter perform. Differences in source confusions involving a 'personal' source, in which they imagined themselves performing an action, were not significantly greater than controls. It could be argued that concrete thought processes in 22q11DS might contribute to deficits in tasks examining mental imagery of action performance. Imagining the experimenter perform an action might be too abstract, in comparison to repeating an action statement. However, the nature of the 22q11DS group's confusions between repeated and experimenter items in both directions (mistaking mentally repeated actions for imagine experimenter actions, but also mistaking experimenter actions for mentally repeated actions) suggests that source confusions are more pronounced in 'external' source monitoring conditions. Furthermore, concrete thought processes may also characterize the adolescents matched for IQ 
(IQcontrol group), who do not exhibit such source confusions. Therefore, the performances on the action monitoring task suggest that 'external' source confusions are characteristic of adolescents with 22q11DS. Unexpectedly, the external attribution bias, consisting in recalling as experimenter-performed actions that had been imagined as performed by the self, was not observed in the 22q11DS group. We first discuss how experimental procedures and symptom profile may contribute to the present findings. Second, we discuss how these results may be articulated with current cognitive models. Finally, we conclude by discussing how cerebral alterations in 22q11DS, namely hippocampal volume reduction, may be involved in source monitoring skills.

The action monitoring task used in this study, which made use of material generated exclusively by the participant, reproduced part of the experimental procedure from Larøi et al. (2005). The present results do not replicate the findings obtained with hallucination-prone college students, who were more likely to attribute to the experimenter actions that they had imagined themselves perform, thus exhibiting the characteristic external attribution bias. Discrepancies between the two studies may be accounted for by differences in the experimental procedures, which excluded the actual miming performance of an action in this study. The present paradigm included a unique 'personal' condition involving the self, which may have increased its distinctiveness in comparison to the two 'exterior' conditions, thus favouring source monitoring performances on 'personal' trials (Johnson et al. 1993). Age differences between the participants from both studies must also be considered. This is especially relevant to individuals with 22q11DS, who report schizotypal manifestations more frequently as they get older, reaching around $80 \%$ of the individuals in their late teens (Baker \& Skuse, 2005). It is also possible that source monitoring confusions appear earlier in development, as observed in the current study, and then only polarize themselves around an external attribution during the transition between adolescence and adulthood. Finally, it is possible that external attribution biases are specifically related to hallucination proneness, which could not be measured specifically in the present study. Indeed, we cannot rule out that a more homogeneous group of adolescents recruited specifically for hallucination proneness or expression would exhibit external attribution biases. It should be noted that internal source monitoring paradigms used with symptomatic populations are not systematically associated with external attribution biases. Although two studies (Franck et al. 2000; Larøi et al. 2005) found evidence for such a bias in schizophrenic patients and hallucination-prone college students, Henquet et al. (2005) failed to present such evidence using an internal monitoring paradigm with schizophrenic subjects. Similarly, the adolescents with 22q11DS enrolled in the current study showed no evidence of exaggerated external attribution for 'personal' events. Therefore, although the current findings do not suggest external attribution biases during monitoring of internally generated events in adolescents, they do indicate the presence of increased source monitoring confusions for action monitoring involving 'exterior' sources in high risk for schizophrenia adolescents with 22q11DS.

One of the limitations of this study was to use a schizotypy scale (SPQ), which does not yield a score for hallucination proneness. Unlike previous reports on source monitoring specifically recruiting individuals with increased hallucination proneness (Larøi et al. 2004), or participants with schizophrenia still experiencing hallucinations (Brébion et al. 2000; Franck et al. 2000), the present study involved adolescents with heterogeneous expressions of schizotypal dimensions. As with schizophrenia, schizotypy is a construct that relates to a triad of symptom dimensions (Rossi \& Daneluzzo, 2002) and, as such, can be fairly heterogeneous in terms of its expression in both clinical and non-clinical populations (Kendler et al. 1994). This heterogeneity in symptom profile may also be characteristic of cognitive performances (Joyce \& Roiser, 2007). The cognitive-perceptual subscale of the SPQ is itself heterogeneous, as it includes not only domains of auditory and visual hallucinations but also those of magical thinking, ideas of reference and suspiciousness. Thus, we may not exclude the possibility that heterogeneity of the measures used may conceal finer associations between source memory performances and hallucination proneness during adolescence, which could not be evidenced in our correlational analyses.

Along these lines, we can interpret the performances of the 22q11DS and IQcontrol groups. In the 22q11DS group, increased scores on the interpersonal schizotypy subscale score (which translates excessive social anxiety, constricted affect and social isolation) suggest that 'negative schizotypy' may contribute to source monitoring confusions. This is consistent with a recent study suggesting that adolescents with high negative or mixed schizotypy profiles were related to poor source monitoring performances, especially in the high psycho-social risk group (Lemos Giraldez et al. 2000). Concerning group comparisons between IQcontrols and AGEcontrols, increased levels of positive schizotypy and depression could be observed in the cognitively impaired controls. However, no significant differences were found in their source monitoring performances, except for 
trend-like differences in confusing repeated actions as though they were imagine-self (see Fig. 1 rep_self column). While this may be surprising, we must consider the impact of depressive symptoms in the IQcontrols, which may exert an increased influence on their source monitoring performance at this stage. Indeed, literature on memory performance in depression suggests that depressed individuals display a preferential bias for self-related information (Taylor \& John, 2004). Our instruments also limit the characterization of specific proneness to hallucinations in this group. These considerations point to the necessity of using assessment tools sensitive to differences between hallucination types and delusion ideations. Finally, it should be noted that, to the best of our knowledge, there are no data directly correlating positive symptom intensity and source monitoring memory deficits in the current literature, which further stresses the importance of sensitivity in both the assessment instruments used and the source monitoring paradigms.

The theoretical implications of the current results and their potential neuroanatomical underpinnings remain to be considered. Cognitive models of psychosis draw upon cognitive biases such as source monitoring deficits to explain the psychological phenomena of positive symptoms in schizophrenia (Frith, 1992; Freeman et al. 2002). In particular, Frith (1992) suggests that self-monitoring deficits may yield uncertainty in determining the source of mental events, and lead to feelings of alien control. Conversely, feelings of persecution and paranoia may be associated with deficits in the monitoring of people's intentions, involving reality monitoring skills (Frith \& Done, 1989; Frith, 1992). Results from the action monitoring task suggest that internal representations of 'exterior' sources are prone to confusions in adolescents with 22q11DS. Further research is needed to assess how these confusions contribute to the development of delusional schemas and abnormal perceptual experiences reported by these adolescents, who report both delusions and auditory hallucinations in childhood and adolescence (Baker \& Skuse, 2005; Debbané et al. 2006a), and high rates of schizophrenia during adulthood (Murphy et al. 1999).

From a neuroanatomical standpoint, source monitoring deficits may also relate to hippocampal volume reduction reported in 22q11DS (Debbané et al. 2006b). Functionally, the hippocampus helps in binding an item to its contextual information, namely source information (Yonelinas, 2001). Inconsistencies in binding actions with source information may be related to hippocampal alterations in 22q11DS, and increase potential for source attribution confusions. The action monitoring of 'personal' events may be encoded from a first-person perspective, which further benefits from mesial prefrontal cortex, posterior cingulate cortex and superior temporal cortex activity (Vogeley et al. 2004), regions that are all relatively spared in cerebral development of youngsters with 22q11DS (Kates et al. 2001; Simon et al. 2005b). On the contrary, faulty binding from a third-person perspective, presumably corresponding to the 'exterior' event conditions, may rely on other regions such as the mesial superior parietal and right premotor cortex (Vogeley et al. 2004), but in the case of 22q11DS, midline and parietal cerebral alterations associated with the neurogenetic syndrome (Simon et al. 2005a) may hinder efficient processing in these conditions. Consequently, cerebral alterations linked to the microdeletion on chromosome 22 may contribute to increased source monitoring deficits involving 'exterior' events. These results are preliminary, and further investigations involving structural and functional magnetic resonance imaging are needed to determine the extent to which cerebral structure alterations impede source monitoring functions in this population.

In conclusion, we find that similarly to adults with schizophrenia, adolescents affected by 22q11DS exhibit source monitoring deficits. These monitoring confusions do not correlate directly with schizotypal manifestations, but may set the stage for further development of psychotic symptomatology (Freeman et al. 2002). While source monitoring deficits constitute characteristic memory deficits observed in diagnosed schizophrenia (Bentall et al. 1991; Vinogradov et al. 1997), it appears that such deficits can be found in adolescents at very high risk for schizophrenia. Further examination of the complex relationships between genetic risk for psychosis, cerebral development, source monitoring and psychotic symptoms is necessary to better understand the unfolding of psychosis in 22q11DS and other high-risk adolescent populations. This may provide information on the different pathways leading to the illness of schizophrenia, and may assist in the elaboration of preventive strategies with adolescents at high risk for schizophrenia.

\section{Acknowledgements}

We thank all the participants who kindly volunteered for this study. We extend our special thanks to Frank Larøi for consultations on the experimental design, and to Anne-Laure Bortoli, Christelle Crépin and Sylvie Güller for help in data collection. This work was supported by research grants to Stephan Eliez from the Swiss National Fund for Research (PP00B-102864), the NARSAD Foundation (2002 Lieber Investigator Award) and the Academic Society of Geneva. 


\section{Declaration of Interest}

None.

\section{References}

Allen PP, Johns LC, Fu CH, Broome MR, Vythelingum GN, McGuire PK (2004). Misattribution of external speech in patients with hallucinations and delusions. Schizophrenia Research 69, 277-287.

Axelrod SR, Grilo CM, Sanislow C, McGlashan TH (2001). Schizotypal Personality Questionnaire-Brief: factor structure and convergent validity in inpatient adolescents. Journal of Personality Disorders 15, 168-179.

Baker KD, Skuse DH (2005). Adolescents and young adults with 22q11 deletion syndrome: psychopathology in an at-risk group. British Journal of Psychiatry 186, 115-120.

Bassett AS, Chow EW, AbdelMalik P, Gheorghiu M, Husted J, Weksberg R (2003). The schizophrenia phenotype in 22q11 deletion syndrome. American Journal of Psychiatry 160, 1580-1586.

Bentall RP, Baker GA, Havers S (1991). Reality monitoring and psychotic hallucinations. British Journal of Clinical Psychology 30, 213-222.

Bentall RP, Kinderman P, Kaney S (1994). The self, attributional processes and abnormal beliefs: towards a model of persecutory delusions. Behaviour Research and Therapy 32, 331-341.

Brébion G, Amador X, David A, Malaspina D, Sharif Z, Gorman JM (2000). Positive symptomatology and source-monitoring failure in schizophrenia - an analysis of symptom-specific effects. Psychiatry Research 95, 119-131.

Brébion G, Gorman JM, Amador X, Malaspina D, Sharif $\mathbf{Z}$ (2002). Source monitoring impairments in schizophrenia: characterisation and associations with positive and negative symptomatology. Psychiatry Research 112, 27-39.

Brébion G, Gorman JM, Malaspina D, Amador X (2005). A model of verbal memory impairments in schizophrenia: two systems and their associations with underlying cognitive processes and clinical symptoms. Psychological Medicine 35, 133-142.

Brunelin J, Combris M, Poulet E, Kallel L, D'Amato T, Dalery J, Saoud M (2006). Source monitoring deficits in hallucinating compared to non-hallucinating patients with schizophrenia. European Psychiatry 21, 259-261.

Cornblatt B, Obuchowski M, Roberts S, Pollack S, Erlenmeyer-Kimling L (1999). Cognitive and behavioral precursors of schizophrenia. Developmental Psychopathology 11, 487-508.

Debbané M, Glaser B, David MK, Feinstein C, Eliez S (2006a). Psychotic symptoms in children and adolescents with 22q11.2 deletion syndrome: neuropsychological and behavioral implications. Schizophrenia Research 84, 187-193.

Debbané M, Glaser B, Eliez S (in press). Encoding and retrieval processes in velo-cardio-facial syndrome (VCFS). Neuropsychology.
Debbané M, Schaer M, Farhoumand R, Glaser B, Eliez S (2006b). Hippocampal volume reduction in 22q11.2 deletion syndrome. Neuropsychologia 44, 2360-2365.

Dumas P, Bouafia S, Gutknecht C, Saoud M, Dalery J, d'Amato T (2000). Validation of the French version of the Raine Schizotypal Personality Disorder Questionnaire - categorical and dimensional approach to schizotypal personality traits in a normal student population [in French]. Encephale 26, 23-29.

Franck N, Rouby P, Daprati E, Dalery J, Marie-Cardine M, Georgieff N (2000). Confusion between silent and overt reading in schizophrenia. Schizophrenia Research 41, 357-364.

Freeman D, Garety PA, Kuipers E, Fowler D, Bebbington PE (2002). A cognitive model of persecutory delusions. British Journal of Clinical Psychology 41, 331-347.

Frith C, Done J (1989). Positive symptoms of schizophrenia. British Journal of Psychiatry 154, 569-570.

Frith CD (1992). The Cognitive Neuropsychology of Schizophrenia. Psychology Press Ltd: Hove.

Glaser B, Mumme DL, Blasey C, Morris MA, Dahoun SP, Antonarakis SE, Reiss AL, Eliez S (2002). Language skills in children with velocardiofacial syndrome (deletion 22q11.2). Journal of Pediatrics 140, 753-758.

Golding-Kushner KJ, Weller G, Shprintzen RJ (1985). Velo-cardio-facial syndrome: language and psychological profiles. Journal of Craniofacial Genetics and Developmental Biology 5, 259-266.

Gothelf D, Eliez S, Thompson T, Hinard C, Penniman L, Feinstein C, Kwon H, Jin S, Jo B, Antonarakis SE, Morris MA, Reiss AL (2005). COMT genotype predicts longitudinal cognitive decline and psychosis in 22q11.2 deletion syndrome. Nature Neuroscience 8, 1500-1502.

Henquet C, Krabbendam L, Dautzenberg J, Jolles J, Merckelbach H (2005). Confusing thoughts and speech: source monitoring and psychosis. Psychiatry Research 133, 57-63.

Johns LC, van Os J (2001). The continuity of psychotic experiences in the general population. Clinical Psychology Review 21, 1125-1141.

Johnson MK, Hashtroudi S, Lindsay DS (1993). Source monitoring. Psychological Bulletin 114, 3-28.

Johnson MK, Raye CL (2000). Cognitive and brains mechanisms of false memories and beliefs. In Memory, Brain and Belief (ed. D. L. Schacter and E. Scarry), pp. 35-86. Harvard University Press: Cambridge, MA.

Joyce EM, Roiser JP (2007). Cognitive heterogeneity in schizophrenia. Current Opinion in Psychiatry 20, 268-272.

Kates WR, Burnette CP, Jabs EW, Rutberg J, Murphy AM, Grados M, Geraghty M, Kaufmann WE, Pearlson GD (2001). Regional cortical white matter reductions in velocardiofacial syndrome: a volumetric MRI analysis. Biological Psychiatry 49, 677-684.

Kaufman AS, Kaufman JC, Balgopal R, McLean JE (1996). Comparison of three WISC-III short-forms: weighing psychometric, clinical, and practical factors. Journal of Clinical Child Psychology 25, 97-105. 
Kendler KS, McGuire M, Gruenberg AM, Walsh D (1994). Clinical heterogeneity in schizophrenia and the pattern of psychopathology in relatives: results from an epidemiologically based family study. Acta Psychiatrica Scandinavica 89, 294-300.

Larøi F, Collignon O, Van der Linden M (2005). Source monitoring for actions in hallucination proneness. Cognitive Neuropsychiatry 10, 105-123.

Larøi F, Van der Linden M, Marczewski P (2004). The effects of emotional salience, cognitive effort and meta-cognitive beliefs on a reality monitoring task in hallucination-prone subjects. British Journal of Clinical Psychology 43, 221-233.

Lemos Giraldez S, Caro MI, Lopez Rodrigo AM, Pineiro MP, Gonzalez JL (2000). Assessment of essential components of schizotypy using neurocognitive measures. Psychology in Spain 4, 183-194.

Lewandowski KE, Shashi V, Berry PM, Kwapil TR (2006). Schizophrenic-like neurocognitive deficits in children and adolescents with 22q11 deletion syndrome. American Journal of Medical Genetics. Part B, Neuropsychiatric Genetics 144, 27-36.

Moss EM, Batshaw ML, Solot CB, Gerdes M, McDonaldMcGinn DM, Driscoll DA, Emanuel BS, Zackai EH, Wang PP (1999). Psychoeducational profile of the 22q11.2 microdeletion: a complex pattern. Journal of Pediatrics 134, 193-198.

Murphy KC (2005). Annotation: velo-cardio-facial syndrome. Journal of Child Psychology and Psychiatry 46, 563-571.

Murphy KC, Jones LA, Owen MJ (1999). High rates of schizophrenia in adults with velo-cardio-facial syndrome. Archives of General Psychiatry 56, 940-945.

Oskarsdottir S, Vujic M, Fasth A (2004). Incidence and prevalence of the 22q11 deletion syndrome: a population-based study in Western Sweden. Archives of Disease in Childhood 89, 148-151.

Raine A (1991). The SPQ: a scale for the assessment of schizotypal personality based on DSM-III-R criteria. Schizophrenia Bulletin 17, 555-564.

Rossi A, Daneluzzo E (2002). Schizotypal dimensions in normals and schizophrenic patients: a comparison with other clinical samples. Schizophrenia Research 54, 67-75.

Saint-Laurent L (1990). Psychometric study of Kovacs' Children's Depression Inventory with a French-speaking sample [in French]. Canadian Journal of Behavioral Science 22, 377-384.

Simon TJ, Bish JP, Bearden CE, Ding L, Ferrante S, Nguyen V, Gee JC, McDonald-McGinn DM, Zackai EH, Emanuel BS (2005a). A multilevel analysis of cognitive dysfunction and psychopathology associated with chromosome 22q11.2 deletion syndrome in children. Developmental Psychopathology 17, 753-784.

Simon TJ, Ding L, Bish JP, McDonald-McGinn DM, Zackai EH, Gee J (2005b). Volumetric, connective, and morphologic changes in the brains of children with chromosome 22q11.2 deletion syndrome: an integrative study. Neuroimage 25, 169-180.

Swillen A, Devriendt K, Legius E, Eyskens B, Dumoulin M, Gewillig M, Fryns JP (1997). Intelligence and psychosocial adjustment in velocardiofacial syndrome: a study of 37 children and adolescents with VCFS. Journal of Medical Genetics 34, 453-458.

Swillen A, Vandeputte L, Cracco J, Maes B, Ghesquiere P, Devriendt K, Fryns JP (1999). Neuropsychological, learning and psychosocial profile of primary school aged children with the velo-cardio-facial syndrome (22q11 deletion): evidence for a nonverbal learning disability? Child Neuropsychology 5, 230-241.

Taylor JL, John CH (2004). Attentional and memory bias in persecutory delusions and depression. Psychopathology 37, 233-241.

Turgeon L, Chartrand E (2003). Reliability and validity of the revised children's manifest anxiety scale in a French-Canadian sample. Psychological Assessment 15, 378-383.

Vinogradov S, Willis-Shore J, Poole JH, Marten E, Ober BA, Shenaut GK (1997). Clinical and neurocognitive aspects of source monitoring errors in schizophrenia. American Journal of Psychiatry 154, 1530-1537.

Vogeley K, May M, Ritzl A, Falkai P, Zilles K, Fink GR (2004). Neural correlates of first-person perspective as one constituent of human self-consciousness. Journal of Cognitive Neuroscience 16, 817-827.

Yonelinas AP (2001). Components of episodic memory: the contribution of recollection and familiarity. Philosophical Transactions of the Royal Society of London. Series B, Biological Sciences 356, 1363-1374. 